\title{
Reversed time in Mössbauer time spectra
}

\author{
Yu.V. Shvyd'ko* \\ Russian Research Centre "Kurchatov Institute," 123182, Moscow, Russia \\ T. Hertrich \\ Physik-Department E15, Technische Universität München, D-85748, Garching, Germany \\ J. Metge, O. Leupold, E. Gerdau, and H.D. Rüter \\ II. Institut für Experimentalphysik, Universität Hamburg, D-22761, Hamburg, Germany
}

(Received 13 March 1995)

\begin{abstract}
Mössbauer time spectra were studied under the condition of abrupt inversion of the hyperfine magnetic fields. The 14.4-keV nuclear resonance of ${ }^{57} \mathrm{Fe}$ in an ${ }^{57} \mathrm{FeBO}_{3}$ crystal was excited by synchrotron radiation pulses in Bragg-diffraction conditions. Fast inversion of the crystal magnetization and the hyperfine magnetic fields causes the time reversal of the quantum-beat pattern in the Mössbauer time spectra.
\end{abstract}

The power of any spectroscopic method is greatly enhanced if the various types of line broadening can be resolved or eliminated. High-resolution variants of NMR, optical, and neutron spectroscopies achieve line narrowing by observing time-reversal or echo phenomena. ${ }^{1-3}$ Such techniques could be developed in Mössbauer spectroscopy as well if one were able to start up a time-reversal process during the lifetime of excited Mössbauer nuclei. Their excitation energies typically lie in the $10-\mathrm{keV}$ range. Therefore, it is difficult to apply classical methods of time reversal as high power radiation fields are required (to induce Rabi oscillations between the ground and excited nuclear states). In this paper an alternative possibility is considered and realized experimentally in nuclear resonant scattering of photons. We demonstrate that via the magnetic interaction of nuclei with ambient fields it is possible to start up time reversal by fast inversion of these fields.

The Hamiltonian of a nucleus in a crystal $n$ site with energy $E_{\lambda}^{(0)}$, spin $I_{\lambda}$, and magnetic moment $\mu_{\lambda}$ in the ground $(\lambda=g)$ or in the excited state $(\lambda=e)$ which experiences the magnetic field $\boldsymbol{B}^{(n)}$ is given by

$$
\hat{\mathscr{H}}_{\lambda}^{(n)}=\hat{\mathscr{H}}_{\lambda}^{(0)}-\mu_{\lambda} \frac{\hat{\boldsymbol{I}}_{\lambda} \cdot \boldsymbol{B}^{(n)}}{I_{\lambda}} .
$$

According to the rules of quantum mechanics ${ }^{4}$ the operation of time reversal $\hat{K}$ inverts the spin $I_{\lambda}$ and transforms its eigenstates $\left|m_{\lambda}\right\rangle$ to the complex-conjugate ones

$$
\hat{K} \hat{\boldsymbol{I}}_{\lambda} \hat{K}^{\dagger}=-\hat{\boldsymbol{I}}_{\lambda}, \quad \hat{K}\left|m_{\lambda}\right\rangle=\left|m_{\lambda}\right\rangle^{*} .
$$

Therefore for the part of the Hamiltonian which takes into account the interaction of the nuclear spin $I_{\lambda}$ with the magnetic field $\boldsymbol{B}^{(n)}$ the inversion $\boldsymbol{B}^{(n)} \Rightarrow-\boldsymbol{B}^{(n)}$ produces the same effect as the operation of time reversal-Eq. (2). This property is used in the present studies to "reverse" time in Mössbauer time spectra.

First we consider theoretically the scattering problem pertinent to our experiment. The nuclei in a crystal which experience time-dependent hyperfine interactions are excited at $t=0$ by a short pulse of $\gamma$ radiation in an $e^{s}(s=\pi, \sigma)$ po- larization state. The amplitude $E_{\nu}(t)$ of the radiation reemitted coherently in the forward $(\nu=0)$ or in the Bragg $(\nu=1)$ directions at time $t$ by the excited nuclei is given in the Born approximation by the expression

$$
\boldsymbol{E}_{\nu}(t) \propto \sum_{\psi_{g}, \psi_{e}, n} e^{i \boldsymbol{K}_{\nu} \boldsymbol{\rho}^{(n)}} J_{\psi_{g} \psi_{e}}^{(n)}\left(\boldsymbol{k}_{\nu}, t\right) J_{\psi_{e} \psi_{g}}^{s(n)}\left(-\boldsymbol{k}_{0}, 0\right)
$$

Here $J_{\psi_{g} \psi_{e}}^{(n)}\left(k_{\nu}, t\right)$ are the transition matrix elements of the vector operator $\hat{J}^{(n)}\left(\boldsymbol{k}_{\nu}, t\right)=\hat{U}_{\lambda}^{(n) \dagger}(t, 0) \hat{j}^{(n)}\left(\boldsymbol{k}_{\nu}\right) \hat{U}_{\lambda}^{(n)}(t, 0)$ between the ground and excited nuclear states $\left|\psi_{\lambda}\right\rangle$ with $\hat{j}^{(n)}\left(\boldsymbol{k}_{\nu}\right)$ being the nuclear current density vector operator in momentum representation; $\hat{U}_{\lambda}^{(n)}\left(t_{2}, t_{1}\right)=\hat{T} \exp [-(i)$ ћ) $\left.\int_{t_{1}}^{t_{2}} d \tau \hat{\mathscr{H}}_{\lambda}^{(n)}(\tau)\right]$ the evolution, and $\hat{T}$ the time-ordering operators; the nuclear Hamiltonian $\hat{\mathscr{H}}_{\lambda}^{(n)}(t)$ takes into account the time dependence of the hyperfine interactions; the index $s$ in $J_{\psi_{e} \psi_{g}}^{s(n)}\left(-k_{0}, 0\right)$ denotes a component of the vector matrix element along the direction of the polarization vector $\boldsymbol{e}^{s} ; \boldsymbol{k}_{0}$ and $\boldsymbol{k}_{1}$ are the wave vectors of the radiation propagating in the forward and in the Bragg direction, respectively; $\boldsymbol{K}_{\nu}=\boldsymbol{k}_{\nu}-\boldsymbol{k}_{0}$ is a reciprocal lattice vector; $\boldsymbol{\rho}^{(n)}$ is the relative position of a nucleus in the $n$-site of the crystal unit cell.

The nuclear Hamiltonian $\hat{\mathscr{H}}_{\lambda}^{(n)}(t)$ in the specific case of the abrupt inversion of the hyperfine magnetic field at a time instant $t_{\text {sw }}$ can be defined by Eq. (1), however, with the magnetic field being an explicit function of time: $B^{(n)} \Rightarrow B^{(n)}(t)=B^{(n)} \eta\left(t-t_{\mathrm{sw}}\right), \quad$ where $\quad \eta\left(t-t_{\mathrm{sw}}\right)=1$ for $t \leqslant t_{\mathrm{sw}}$ and $\eta\left(t-t_{\mathrm{sw}}\right)=-1$ otherwise. Prior to the inversion $\left(t \leqslant t_{\mathrm{sw}}\right)$ the nuclear Hamiltonian is time independent and is given exactly by Eq. (1). The nuclear states $\left|\psi_{\lambda}\right\rangle$ can be chosen as eigenvectors $\left|m_{\lambda}\right\rangle$ of the spin operator $\hat{I}_{\lambda}$. Then the nuclear transition currents are

$$
\boldsymbol{J}_{m_{g^{m}} m_{e}}^{(n)}\left(\boldsymbol{k}_{\nu}, t\right)=\boldsymbol{j}_{m_{g^{m}} m_{e}}^{(n)}\left(\boldsymbol{k}_{\nu}\right) \exp \left[-i \Omega_{m_{g^{m}}}^{(n)} t\right] \zeta(t)
$$




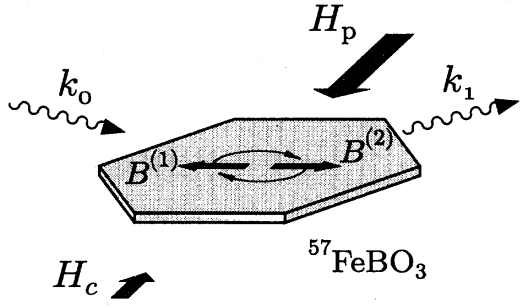

FIG. 1. The time spectra of the nuclear scattering of 14.4-keV synchrotron radiation pulses (Fig. 2) were studied by a pure nuclear Bragg reflection (333) in an antiferromagnetic crystal of ${ }^{57} \mathrm{FeBO}_{3}$ under the following conditions. A constant magnetic field $\boldsymbol{H}_{\mathrm{c}} \perp\left(\boldsymbol{k}_{0}, \boldsymbol{k}_{1}\right)$ aligned the magnetic hyperfine fields $\boldsymbol{B}^{(1)}$ and $\boldsymbol{B}^{(2)}$ in both nuclear sites in the unit cell of the ${ }^{57} \mathrm{FeBO}_{3}$ crystal parallel to $\boldsymbol{k}_{0}+\boldsymbol{k}_{1}$. The crystal magnetization and the hyperfine fields were inverted abruptly by the application of a pulsed magnetic field $\boldsymbol{H}_{p}$ with some delay after the arrival of the synchrotron radiation pulse on the crystal.

where $\zeta(t)=\exp \left(-i E_{0} t / \hbar-\Gamma_{0} t / 2 \hbar\right)$ with $E_{0}=E_{e}^{(0)}-E_{g}^{(0)}$ being the energy and $\Gamma_{0}$ being the full width of the nuclear transition $e \rightarrow g$; while $\hbar \Omega_{m_{g} m_{e}}^{(n)}=\left(\mu_{g} m_{g} / I_{g}-\mu_{e} m_{e} / I_{e}\right) B^{(n)}$ are the relative transition energies arising from the hyperfine magnetic interaction. Insertion of Eq. (4) in Eq. (3) yields the amplitude of the radiation reemitted by the excited nuclei

$$
\begin{gathered}
\boldsymbol{E}_{\nu}(t) \propto \zeta(t) \sum_{m_{g}, m_{e}, n} \mathscr{C}_{\nu}^{(n)}\left(m_{g}, m_{e}\right) \exp \left[-i \Omega_{m_{g} m_{e}}^{(n)} t\right], \\
\mathscr{C}_{\nu}^{(n)}\left(m_{g}, m_{e}\right)=e^{i \boldsymbol{K}_{\nu} \cdot \boldsymbol{\rho}^{(n)}} \boldsymbol{j}_{m_{g} m_{e}}^{(n)}\left(\boldsymbol{k}_{\nu}\right) j_{m_{e} m_{g}}^{s(n)}\left(-\boldsymbol{k}_{0}\right) .
\end{gathered}
$$

These equations describe a typical quantum-beat time spectrum brought about by the interference of all excited hyperfine nuclear transitions after $t>0 .^{5,6}$

The explicit calculations ${ }^{7}$ show that after the instantaneous inversion of the magnetic hyperfine fields $\left(t=t_{\mathrm{sw}}>0\right)$ the transition currents and the time spectrum can be described by the same Eqs. (4)-(6), however, with the following changes:

$$
\begin{gathered}
\boldsymbol{j}_{m_{g} m_{e}}^{(n)}\left(\boldsymbol{k}_{\nu}\right) \Rightarrow \boldsymbol{j}_{m_{g} m_{e}}^{(n) *}\left(-\boldsymbol{k}_{\nu}\right), \\
\exp \left[-i \Omega_{m_{g^{m}} m_{e}}^{(n)} t\right] \Rightarrow \exp \left[-i \Omega_{m_{g} m_{e}}^{(n)}\left(2 t_{\mathrm{sw}}-t\right)\right] .
\end{gathered}
$$

This result is expected from the general rules of time reversal-Eq. (2). We can conclude now that the hyperfine magnetic field inversion indeed reverses the time evolution of the relative phases of the nuclear transition currents. [There is no such effect on the factor $\zeta(t)$ common for all hyperfine transitions.] As a consequence the relative phases of all the nuclear transition currents will return to their initial values at the time instant $t=2 t_{\mathrm{sw}}$.

To bring off this mechanism of time reversal one needs samples that allow the inversion of the hyperfine magnetic field direction within a time shorter than both the nuclear lifetime $\tau_{0}$ and the periods of the Larmor precession $\tau_{\lambda}=2 \pi \hbar I_{\lambda} /\left(\mu_{\lambda} B^{(n)}\right), \lambda=g, e$. These conditions could be met for nuclei with long-lived excited states $\tau_{0} \geqslant 10^{-6} \mathrm{~s}$, e.g.,
${ }^{181} \mathrm{Ta},{ }^{67} \mathrm{Zn},{ }^{45} \mathrm{Sc}$, etc. These conditions could be met well also for ${ }^{57} \mathrm{Fe}$ - the standard Mössbauer isotope, since $\mathrm{Fe}$ is the main component in a number of soft magnetic materials ${ }^{8-10}$ which allow easily to rotate the magnetization and the direction of the hyperfine magnetic fields.

For demonstration of the time reversal effect the ${ }^{57} \mathrm{Fe}$ nuclei in an ${ }^{57} \mathrm{FeBO}_{3}$ crystal $\left(E_{0}=14.4 \mathrm{keV}, \tau_{0}=141 \mathrm{~ns}\right.$, $\tau_{\lambda} \geqslant 25 \mathrm{~ns}$ ) were used. The weak ferromagnet ${ }^{57} \mathrm{FeBO}_{3}$ is known to allow fast rotation of its magnetization. ${ }^{11-13}$ The experiment was performed at the storage ring DORIS III (DESY, Hamburg). ${ }^{14}$ The nuclei were excited by synchrotron radiation pulses. The crystal was set in the position of the pure nuclear Bragg reflection (333)-Fig. 1. Time spectra of the nuclear Bragg diffraction were measured.

A constant external magnetic field $H_{c}=5$ Oe was applied

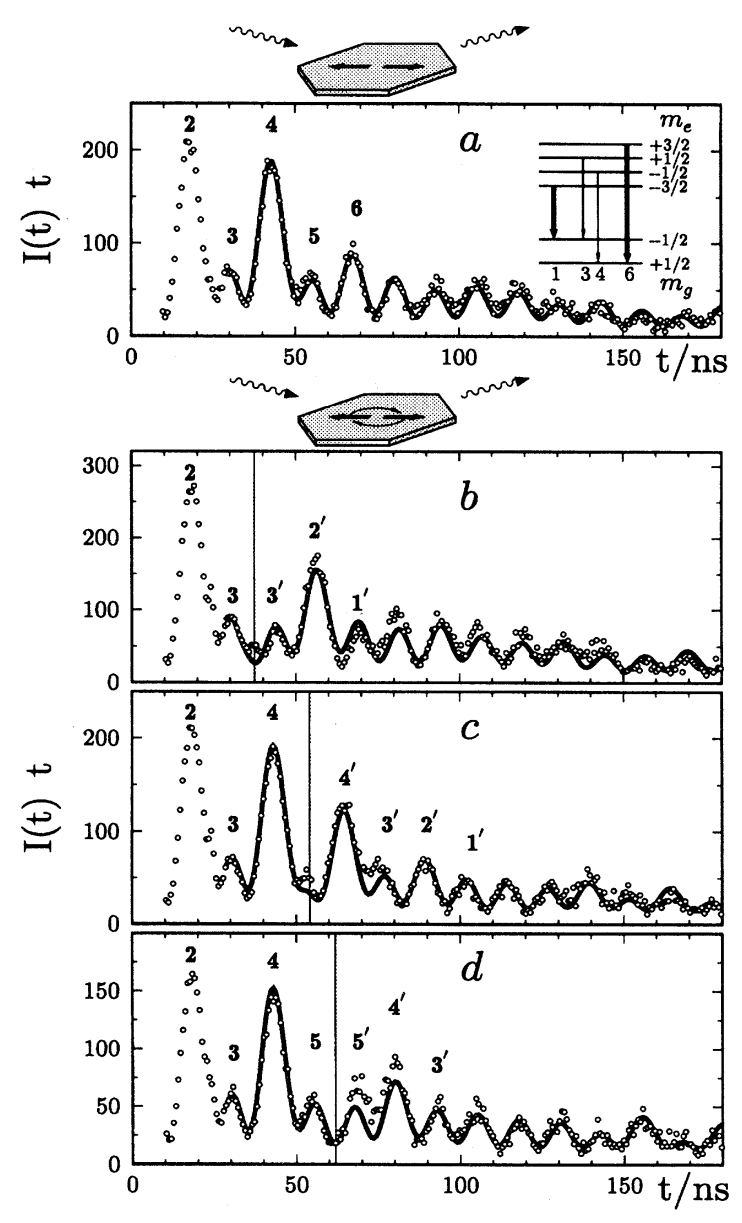

FIG. 2. Mössbauer diffraction time spectra in ${ }^{57} \mathrm{FeBO}_{3}$ (333); scattering geometries with the directions of the hyperfine magnetic fields and level scheme with the corresponding hyperfine nuclear transitions in ${ }^{57} \mathrm{Fe}$ : (a) unperturbed time spectrum; (b)-(d) perturbed time spectra obtained by sudden rotation of the hyperfine fields by $180^{\circ}$ at different instants after exciting the nuclei by the synchrotron radiation pulse. The solid lines are calculated theoretical spectra. The vertical dotted lines indicate the instants of switching $t_{\mathrm{sw}}$ obtained from the fit. These values do not deviate from the experimental ones by more than $\pm 0.6 \mathrm{~ns}$. 
parallel to the surface of the crystal slab and perpendicular to the scattering plane $\left(\boldsymbol{k}_{0}, \boldsymbol{k}_{1}\right)$. This field aligned the magnetic hyperfine fields $\boldsymbol{B}^{(1)}$ and $\boldsymbol{B}^{(2)}$ in both nuclear sites in the unit cell of the $\mathrm{FeBO}_{3}$ crystal with $\boldsymbol{k}_{0}+\boldsymbol{k}_{1}$.

The fast inversion of the crystal magnetization and the hyperfine magnetic fields was achieved by sudden $(\leqslant 4 \mathrm{~ns})$ application of the magnetic field $H_{p} \simeq 22$ Oe antiparallel to $H_{c}$. The pulsed field $H_{p}$ was produced in a magnetization cell which consisted of four single-loop rectangular coils. Each coil was made of a beryllium strip $2 \mathrm{~mm}$ wide, $80 \mathrm{~mm}$ long, and $0.1 \mathrm{~mm}$ thick. Each strip was bent in the middle so that the distance between the straight parallel parts was 0.5 mm. The ${ }^{57} \mathrm{FeBO}_{3}$ platelet which has the dimensions $10 \times 6$ $\times 0.06 \mathrm{~mm}^{3}$ was inserted inside the loops. The current pulses of $4.5 \mathrm{~A}$ with $3 \mathrm{~ns}$ rise time from a pulsed current generator ${ }^{15}$ were fed to each coil via $12.5 \Omega$ cables. Due to the small area and hence inductance of the loops the current pulses were integrated only slightly. The frequent switching of the magnetization and the direct influence of the currents in the strips produce heating of the sample up to $62{ }^{\circ} \mathrm{C}$.

The magnetic pulses were triggered off by every second pulse of the DORIS III bunch clock which is locked in phase with the synchrotron radiation pulses. This procedure gave the possibility to measure simultaneously spectra both unperturbed and perturbed by the hyperfine field inversion.

Figure 2(a) shows the unperturbed time spectrum measured with the fixed directions of the hyperfine fields when only the constant external magnetic field $H_{c}$ was applied. The dominant intensity beats with the period $12.6 \mathrm{~ns}$ arise from the interference of the strongest transitions 1 and 6 (see Ref. 16 for more details).

The spectra in Figs. 2(b)-(d) were measured when additionally the pulsed magnetic field was applied to the crystal with some delay after the arrival of the synchrotron radiation pulse. These time instants are indicated by dotted lines. One can notice that with the allowance for the nuclear decay the spectra after the inversion are the mirror images in time of those before the inversion. That is, in the unperturbed spectrum in Fig. 2(a) the fourth quantum beat maximum is much more pronounced than the third one. On the other hand, in the perturbed spectrum in Fig. 2(b) in place of the fourth quantum beat appears the one, denoted as $3^{\prime}$, with nearly the same intensity as the third one. The quantum beat $3^{\prime}$ can be interpreted as the echo of the quantum beat 3. A similar behavior is seen in the other spectra.

The experimental spectra were consistently fitted by using the theory outlined above, however, with two modifications. The first one takes into account the speeded-up decay of the collective nuclear excitation, which exhibits itself in thick crystals like that one used in the experiment. The time spectra were calculated by using the asymptotic formula of the dynamic theory of diffraction ${ }^{17}$

$$
I(t) \propto \frac{1}{t^{\kappa}}\left|E_{1}(t)\right|^{2}, \quad t \geqslant t^{*}
$$

The damping parameter $\kappa$ can take values in the range $1 \leqslant \kappa \leqslant 3$ depending on direction and divergence of the incident beam. In our particular case it is $\kappa=1.57$. The formula turns out to work well at $t^{*} \geqslant 25 \mathrm{~ns}$.

The second modification takes into account the perturbation of the magnetic hyperfine splitting due to a weak quadrupole interaction of ${ }^{57} \mathrm{Fe}$ nuclei with the electric field gradient in ${ }^{57} \mathrm{FeBO}_{3}$ (see, e.g., Ref. 16 for more details). As a result additional time phase factors $\exp \left(-i \Delta \Omega_{\left|m_{g}\right| \mid m_{e}} t^{t}\right)$ appear in Eqs. (4) and (5) which are insensitive to the hyperfine magnetic field inversion. It is because of the presence of the small quadrupole interaction that the time reversal effect in Figs. 2(b)-(d) is not pure.

The unperturbed spectrum in Fig. 2(a) was fitted by using Eqs. (5), (6), and (9) with modifications mentioned above. The spectra in Figs. 2(b)-(d) were fitted by using Eqs. (5)(9). All the nuclear and crystal parameters were taken from the fit of the unperturbed spectra. The only free parameter was the switching time $t_{\mathrm{sw}}$. The switching times obtained from the fit do not deviate from the experimental ones by more than $\pm 0.6 \mathrm{~ns}$.

In the summary, we have investigated the effect of a fast magnetic hyperfine field inversion on the Mössbauer time spectra. The inversion of the field produces the reversal of the quantum-beat pattern in time. It is demonstrated that the hyperfine magnetic field inversion can be used as a start-up mechanism of time reversal in cases where the nuclei experience magnetic hyperfine interactions. This effect could be of particular interest in cases where the nuclear resonances possess a large broadening due to inhomogeneous hyperfine magnetic interaction, e.g., distribution of hyperfine fields in magnetic alloys, spin-spin interactions with neighboring atoms (especially for nuclei with long-lived excited states), etc. The broadening wipes off the coherent signal whereas time reversal can revive it as a $\gamma$-echo signal. The true width of the nuclear resonance obscured by inhomogeneous hyperfine magnetic interactions may be then reconstructed by performing a sequence of hyperfine magnetic field reversals in the sample and by measuring the envelope of the $\gamma$-echo signals. Such reconstruction procedure basically follows the concept of the high-resolution techniques of Carr and Purcell in NMR spectroscopy. ${ }^{18}$

This work has been funded by the Bundesministerium für Forschung und Technologie under Contract Nos. 05 5GUAAI 6 and 05 5WOAAI. Yu.V. Shvyd'ko acknowledges financial support from the Alexander von Humboldt Foundation.

\footnotetext{
${ }^{*}$ Present address: II. Institut für Experimentalphysik, Universität Hamburg, D-22761, Hamburg, Germany.

${ }^{1}$ E.L. Hahn, Phys. Rev. 80, 570 (1950).

${ }^{2}$ I.D. Abella, N.A. Kurnit, and S.R. Hartman, Phys. Rev. 141, 491 (1966).

${ }^{3}$ F. Mezei, Z. Phys. 255, 146 (1972); Neutron Spin Echo, edited by
}

F. Mezei, Lecture Notes in Physics Vol. 128 (Springer-Verlag, Berlin, 1980).

${ }^{4}$ A. Messiah, Quantum Mechanics (North-Holland Publishing, Amsterdam, 1962), Vol. II, p. 669.

${ }^{5}$ G.T. Trammell and J.P. Hannon, Phys. Rev. B 18165 (1978); 19, 3835 (1979). 


\section{RAPID COMMUNICATIONS}

${ }^{6}$ E. Gerdau, R. Rüffer, R. Hollatz, and J.P. Hannon, Phys. Rev. Lett. 57, 1141 (1986).

${ }^{7}$ Yu.V. Shvyd'ko, Hyperfine Interact. 90, 287 (1994).

${ }^{8}$ O.S. Kolotov, V.A. Pogozhev, and R.V. Telesnin, Usp. Fiz. Nauk 113, 569 (1974) [Sov. Phys. Usp. 17, 569 (1975)].

${ }^{9} \mathrm{M}$ Takahashi and T. Shimatsu, IEEE Trans. Magn. 26, 1485 (1990).

${ }^{10}$ H.J. de Wit, Rep. Prog. Phys. 55, 113 (1992).

${ }^{11}$ G.V. Smirnov, Yu.V. Shvyd'ko, O.S. Kolotov, V.A. Pogozhev, M. Kotrbova, S. Kadečkova, and J. Novak, Zh. Eksp. Teor. Fiz. 86, 1495 (1984) [Sov. Phys. JETP 59875 (1984)].

${ }^{12}$ Yu.V. Shvyd'ko, S.L. Popov, and G.V. Smirnov, J. Phys. Condens. Matter 5, 1557 (1993); 5, 7047 (1993).

${ }^{13}$ Yu.V. Shvyd'ko, A.I. Chumakov, G.V. Smirnov, T. Hertrich, U. van Bürck, H.D. Rüter, O. Leupold, J. Metge, and E. Gerdau, Europhys. Lett. 26, 215 (1994).

${ }^{14}$ R. Rüffer, D. Giesenberg, H.D. Rüter, R. Hollatz, E. Gerdau, J. Metge, K. Ruth, W. Sturhahn, M. Grote, and R. Röhlsberger, Hyperfine Interact. 58, 2467 (1990).

${ }^{15}$ O.S. Kolotov, V.A. Pogozhev, G.V. Smirnov, and Yu.V. Shvyd'ko, Prib. Tekh. Eksp. No. 2, 100 (1983) [Instrum. Exp. Tech. (USSR) 26, 347 (1983)].

${ }^{16}$ U, vab Bürck, R.L. Mössbauer, E. Gerdau, R. Rüffer, R. Hollatz, G.V. Smirnov, and J.P. Hannon, Phys. Rev. Lett. 59, 355 (1987).

${ }^{17}$ Yu. Kagan, A.M. Afanas'ev, and V.G. Kohn, J. Phys. C 12, 615 (1979).

${ }^{18}$ C.P. Slichter, Principles of Magnetic Resonance (Springer-Verlag, Berlin, 1978), Chap. 8. 


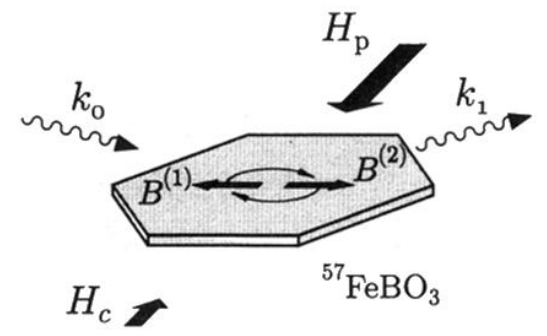

FIG. 1. The time spectra of the nuclear scattering of $14.4-\mathrm{keV}$ synchrotron radiation pulses (Fig. 2) were studied by a pure nuclear Bragg reflection (333) in an antiferromagnetic crystal of ${ }^{57} \mathrm{FeBO}_{3}$ under the following conditions. A constant magnetic field $H_{\mathrm{c}} \perp\left(\boldsymbol{k}_{0}, \boldsymbol{k}_{1}\right)$ aligned the magnetic hyperfine fields $\boldsymbol{B}^{(1)}$ and $\boldsymbol{B}^{(2)}$ in both nuclear sites in the unit cell of the ${ }^{57} \mathrm{FeBO}_{3}$ crystal parallel to $\boldsymbol{k}_{0}+\boldsymbol{k}_{1}$. The crystal magnetization and the hyperfine fields were inverted abruptly by the application of a pulsed magnetic field $\boldsymbol{H}_{p}$ with some delay after the arrival of the synchrotron radiation pulse on the crystal. 

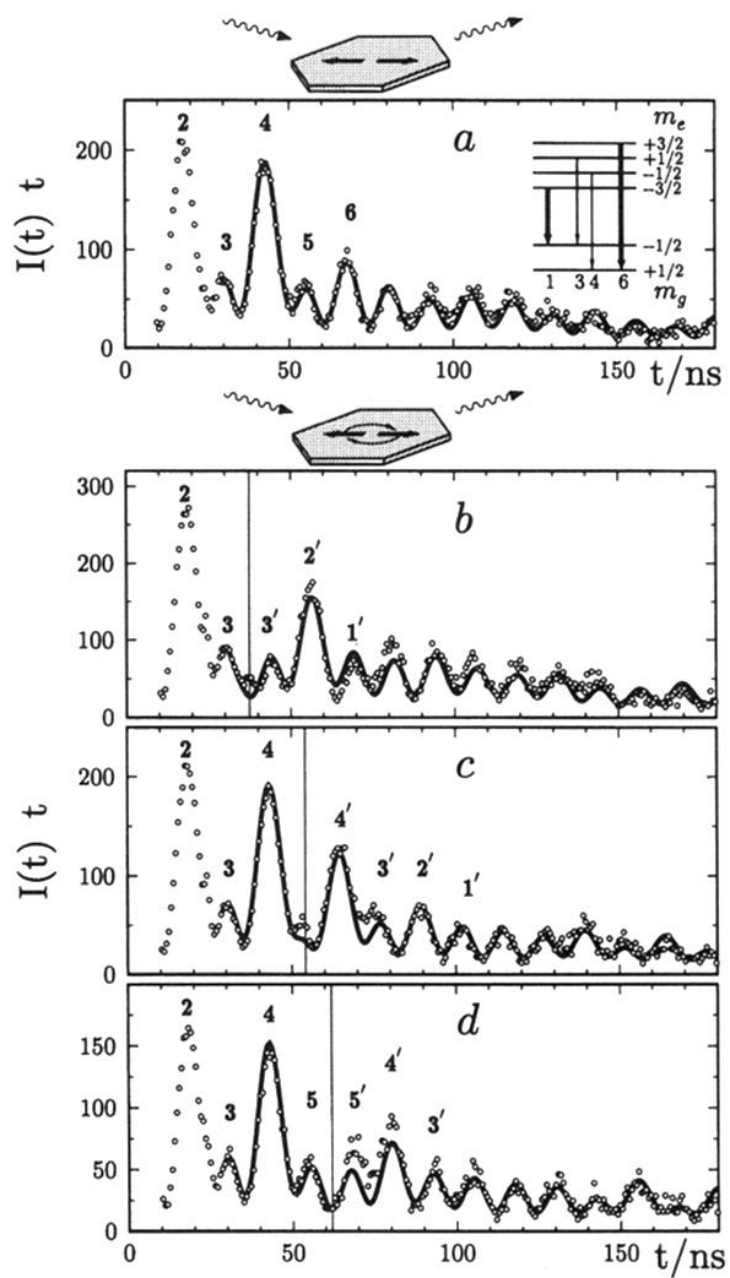

FIG. 2. Mössbauer diffraction time spectra in ${ }^{57} \mathrm{FeBO}_{3}$ (333); scattering geometries with the directions of the hyperfine magnetic fields and level scheme with the corresponding hyperfine nuclear transitions in ${ }^{57} \mathrm{Fe}$ : (a) unperturbed time spectrum; (b)-(d) perturbed time spectra obtained by sudden rotation of the hyperfine fields by $180^{\circ}$ at different instants after exciting the nuclei by the synchrotron radiation pulse. The solid lines are calculated theoretical spectra. The vertical dotted lines indicate the instants of switching $t_{\mathrm{sw}}$ obtained from the fit. These values do not deviate from the experimental ones by more than $\pm 0.6 \mathrm{~ns}$. 